\title{
Complete minimal submanifolds with nullity in Euclidean spheres
}

\author{
M. Dajczer, Th. Kasioumis, A. Savas-Halilaj and Th. Vlachos
}

\begin{abstract}
In this paper we investigate $m$-dimensional complete minimal submanifolds in Euclidean spheres with index of relative nullity at least $m-2$ at any point. These are austere submanifolds in the sense of Harvey and Lawson [19] and were initially studied by Bryant [3]. For any dimension and codimension there is an abundance of non-complete examples fully described by Dajczer and Florit [7] in terms of a class of surfaces, called elliptic, for which the ellipse of curvature of a certain order is a circle at any point. Under the assumption of completeness, it turns out that any submanifold is either totally geodesic or has dimension three. In the latter case there are plenty of examples, even compact ones. Under the mild assumption that the Omori-Yau maximum principle holds on the manifold, a trivial condition in the compact case, we provide a complete local parametric description of the submanifolds in terms of 1-isotropic surfaces in Euclidean space. These are the minimal surfaces for which the standard ellipse of curvature is a circle at any point. For these surfaces, there exists a Weierstrass type representation that generates all simply connected ones.
\end{abstract}

Let $M^{m}$ be a complete $m$-dimensional Riemannian manifold. In [10] we considered the case of minimal isometric immersions into Euclidean space $f: M^{m} \rightarrow \mathbb{R}^{n}, m \geq 3$, satisfying that the index of relative nullity is at least $m-2$ at any point. Under the mild assumption that the Omori-Yau maximum principle holds on $M^{m}$, we concluded that any $f$ must be "trivial", namely, just a cylinder over a complete minimal surface. This result is global in nature since for any dimension there are plenty of non-complete examples other than open subsets of cylinders.

It is natural to expect rather different type of conclusions when considering a similar global problem for minimal isometric immersions into nonflat space forms. For instance, for submanifolds in the hyperbolic space one would guess that under the same condition on the relative nullity index there exist many non-trivial examples, and that a kind of triviality conclusion will only hold under a strong additional assumption.

The third author would like to acknowledge financial support from the grant DFG SM 78/6-1. 
This paper is devoted to the case of minimal submanifolds in round spheres. Thus, in the sequel $f: M^{m} \rightarrow \mathbb{S}^{n}, m \geq 3$, will be a minimal isometric immersion into Euclidean sphere with index of relative nullity at least $m-2$ at any point. As in the Euclidean case, it is known that are plenty of non-complete examples of any dimension. On one hand, under the assumption of completeness of $M^{m}$ we have that $f$ has to be totally geodesic unless $m=3$. On the other hand, for dimension $m=3$ we will see that there are plenty of non trivial examples, even compact ones.

Notice that the class of submanifolds studied in this paper are precisely the minimal $\delta(2)$-ideal submanifolds considered by Chen; see [5].

From the results in [7] it follows that any example for dimension $m=3$ can locally be constructed as follows:

Let $g: L^{2} \rightarrow \mathbb{R}^{n+1}, n \geq 4$, be an elliptic surface whose first curvature ellipse is always a circle. Then, the map $\psi_{g}: T^{1} L \rightarrow \mathbb{S}^{n}$ defined on the unit tangent bundle of $L^{2}$ and given by

$$
\psi_{g}(x, w)=g_{*} w
$$

parametrizes (outside singular points) a minimal immersion $f: M^{3} \rightarrow \mathbb{S}^{n}$ with index of relative nullity at least one at any point.

Minimal surfaces are elliptic, but the latter class of surfaces is much larger. In fact, that a surface $g: L^{2} \rightarrow \mathbb{R}^{n}$ is elliptic means that given a (hence any) basis $X, Y$ of the tangent plane $T_{x} L$ at any $x \in L^{2}$ the second fundamental form $\alpha_{g}: T L \times T L \rightarrow N_{g} L$ of $g$ satisfies

$$
a \alpha_{g}(X, X)+2 b \alpha_{g}(X, Y)+c \alpha_{g}(Y, Y)=0
$$

where $a, b, c \in \mathbb{R}$ verify $a c-b^{2}>0$. Equivalently, in any local system of coordinates $(u, v)$ of $L^{2}$ any coordinate function of $g$ is a solution of the (same) elliptic PDE of type

$$
a \frac{\partial^{2}}{\partial u^{2}}+2 b \frac{\partial^{2}}{\partial u \partial v}+c \frac{\partial^{2}}{\partial v^{2}}+d \frac{\partial}{\partial u}+e \frac{\partial}{\partial v}=0
$$

where $a, b, c, d, e$ are smooth functions such that $a c-b^{2}>0$.

Our main result shows that for complete submanifolds the condition of ellipticity of the generating surface has to be restricted to minimality. For minimal surfaces, the first curvature ellipse as an elliptic surface coincides with the standard ellipse of curvature, namely, the image in the normal space of the second fundamental form restricted to the unit circle in the tangent plane.

Theorem 1. Let $f: M^{m} \rightarrow \mathbb{S}^{n}, m \geq 3$, be a minimal isometric immersion with index of relative nullity at least $m-2$ at any point. If $M^{m}$ is complete then $f$ is totally geodesic unless $m=3$. Moreover, if the Omori-Yau maximum principle holds on $M^{3}$ then along an open dense subset $f$ is locally parametrized by (1) where $g: L^{2} \rightarrow \mathbb{R}^{n+1}$ is a minimal surface whose first curvature ellipse is always a circle. 
A minimal surface $g: L^{2} \rightarrow \mathbb{R}^{n}$ whose first curvature ellipse is a circle at any point is called a 1-isotropic surface. The above result should be complemented by the fact that there is the Weierstrass type representation from [9] that generates all simply connected 1-isotropic surfaces. It goes as follows: start with any nonzero holomorphic map in a simply connected domain $\alpha_{0}: U \subset \mathbb{C} \rightarrow \mathbb{C}^{n-4}$ and let $\alpha_{1}: U \rightarrow \mathbb{C}^{n-2}$ be given by

$$
\alpha_{1}=\beta_{1}\left(1-\phi_{0}^{2}, i\left(1+\phi_{0}^{2}\right), 2 \phi_{0}\right)
$$

where $\phi_{0}=\int^{z} \alpha_{0} d z$ and $\beta_{1} \neq 0$ is any holomorphic function. Now let $\alpha_{2}: U \rightarrow \mathbb{C}^{n}$ be given by

$$
\alpha_{2}=\beta_{2}\left(1-\phi_{1}^{2}, i\left(1+\phi_{1}^{2}\right), 2 \phi_{1}\right)
$$

where $\phi_{1}=\int^{z} \alpha_{1} d z$ and $\beta_{2} \neq 0$ is any holomorphic function. Then $g=\operatorname{Re}\left\{\alpha_{2}\right\}$ is a 1 -isotropic surface in $\mathbb{R}^{n}$.

The Omori-Yau maximum principle holds on $M^{m}$ if for any function $\varphi \in C^{2}(M)$ bounded from above there exists a sequence of points $\left\{x_{j}\right\}_{j \in \mathbb{N}}$ such that

$$
\varphi\left(x_{j}\right)>\sup _{M} \varphi-1 / j, \quad\left\|\nabla \varphi\left(x_{j}\right)\right\| \leq 1 / j \quad \text { and } \quad \Delta \varphi\left(x_{j}\right) \leq 1 / j
$$

for any $j \in \mathbb{N}$. There are fairly general assumptions that imply the validity of the Omori-Yau maximum principle on a Riemannian manifold; see [1]. For instance, it is applicable on complete Riemannian manifolds whose Ricci curvature does not decay fast to $-\infty$.

We see next that there are plenty of complete (even compact) examples of threedimensional minimal submanifolds in spheres with index of relative nullity at least one at any point.

Hopf lifts: If $g: L^{2} \rightarrow \mathbb{C P}^{n}, n \geq 2$, is a substantial holomorphic curve, then the Hopf fibration $\mathcal{H}: \mathbb{S}^{2 n+1} \rightarrow \mathbb{C P}^{n}$ induces a circle bundle $M^{3}$ over $L^{2}$. This lifting induces an immersion $f: M^{3} \rightarrow \mathbb{S}^{2 n+1}$ such that $g \circ \pi=\mathcal{H} \circ f$, where $\pi: M^{3} \rightarrow L^{2}$ is the projection map. Such submanifolds are minimal with index of relative nullity at least one if $n=2$ (see [13]) or $n=3$ (see [21]). Moreover, if $L^{2}$ is compact, then $M^{3}$ is also compact.

Tubes over minimal 2-spheres: Due to the work of Calabi, Chern, Barbosa and others, it is known that minimal 2-spheres in spheres are pseudoholomorphic (isotropic) in substantial even codimension. Calabi [4] proved that any such surface in $\mathbb{S}^{2 n}$ is nicely curved if its area is $2 \pi n(n+1)$, and Barbosa showed [2] that the space of these surfaces is diffeomorphic to $S O(2 n+1, \mathbb{C}) / S O(2 n+1, \mathbb{R})$. According to Proposition 5 below such surfaces produce examples of compact three-dimensional minimal submanifolds in $\mathbb{S}^{2 n}$ with index of relative nullity one.

Among the second family of examples given above, there are the submanifolds produced from pseudoholomorphic surfaces $g: \mathbb{S}^{2} \rightarrow \mathbb{S}^{6}$ with area $24 \pi$ which are holomorphic with respect to the nearly Kaehler structure in $\mathbb{S}^{6}$. For instance, this is the situation 
of the Veronese surface in $\mathbb{S}^{6}$. In this case, the compact submanifolds $M^{3}$ are Lagrangian (also called totally real) in $\mathbb{S}^{6}$; see $[13]$.

Corollary 2. Let $f: M^{3} \rightarrow \mathbb{S}^{6}$ be an isometric immersion with index of relative nullity at least one at any point. Assume that $f$ is Lagrangian with respect to the nearly Kaehler structure in $\mathbb{S}^{6}$. If $M^{3}$ is complete and the Omori-Yau maximum principle holds, then $f$ is locally parametrized by (11) along an open dense subset of $M^{3}$ where $g$ is a 2-isotropic surface in $\mathbb{R}^{6}$ (respectively, $\mathbb{R}^{7}$ ) and $f$ is substantial in $\mathbb{S}^{5}$ (respectively, $\mathbb{S}^{6}$ ).

That the surface $g$ is 2-isotropic means that it is 1-isotropic and that the second ellipse of curvature is also a circle at any point. Hence, in the case of $\mathbb{R}^{6}$ we have that $g$ is congruent to a holomorphic curve in $\mathbb{C}^{3} \equiv \mathbb{R}^{6}$.

It follows from the results in [1] that the universal cover of any of the complete threedimensional submanifolds considered in Theorem 1 admits a one-parameter associated family of isometric immersions of the same type. Moreover, that family is trivial if and only if the (local) generating minimal surface is congruent to a holomorphic curve. We refer to Lotay 21 for a discussion about the existence of such an associated family in the case of yet another family of examples.

\section{The relative nullity foliation}

In this section, we recall basic facts on the relative nullity foliation for submanifolds in space forms to be used in the sequel without further reference.

Let $f: M^{m} \rightarrow \mathbb{Q}_{c}^{n}$ denote an isometric immersion of an $m$-dimensional Riemannian manifold $M^{m}$ into the Euclidean space $\mathbb{R}^{n}(c=0)$ or the unit sphere $\mathbb{S}^{n}(c=1)$.

The relative nullity subspace of $f$ at $x \in M^{m}$ is the tangent subspace given by

$$
\mathcal{D}(x)=\left\{X \in T_{x} M: \alpha_{f}(X, Y)=0 \text { for all } Y \in T_{x} M\right\}
$$

where $\alpha_{f}: T M \times T M \rightarrow N_{f} M$ denotes the second fundamental form of $f$. The dimension $\nu(x)$ of $\mathcal{D}(x)$ is called the index of relative nullity of $f$ at $x \in M^{m}$.

For simplicity, in the sequel we call $k(x)=m-\nu(x)$ the rank of $f$ at $x \in M^{m}$. Notice that $k(x)$ is the rank of the Gauss map of $f$ at $x \in M^{m}$. If $f$ has constant rank on an open subset $U \subset M^{m}$, it is an elementary fact that the relative nullity distribution $\mathcal{D}$ along $U$ is integrable and that the leaves of the relative nullity foliation are totally geodesic submanifolds of $M^{m}$ and under $f$ of $\mathbb{Q}_{c}^{n}$.

Let $U \subset M^{m}$ be an open subset where the index of relative nullity $\nu=s>0$ is constant. The following is a well known fundamental result in the theory of isometric immersions (cf. [6]). 
Proposition 3. Let $\gamma:[0, b] \rightarrow M^{m}$ be a geodesic curve such that $\gamma([0, b))$ is contained in a leaf of relative nullity contained in $U$. Then also $\nu(\gamma(b))=s$.

In the sequel, we decompose any tangent vector field $X$ on $M^{m}$ as $X=X^{v}+X^{h}$ according to the orthogonal splitting $T M=\mathcal{D} \oplus \mathcal{D}^{\perp}$.

The splitting tensor $C: \mathcal{D} \times \mathcal{D}^{\perp} \rightarrow \mathcal{D}^{\perp}$ is given by

$$
C(T, X)=-\nabla_{X}^{h} T=-\left(\nabla_{X} T\right)^{h}
$$

for any $T \in \mathcal{D}$ and $X \in \mathcal{D}^{\perp}$. We also regard $C$ as a map $C: \Gamma(\mathcal{D}) \rightarrow \Gamma\left(\operatorname{End}\left(\mathcal{D}^{\perp}\right)\right)$.

The following differential equations for the endomorphism $C_{T}=C(T, \cdot)$ are a well known easy consequence of the Codazzi equation:

$$
\nabla_{S} C_{T}=C_{T} C_{S}+C_{\nabla_{S} T}+c\langle T, S\rangle I
$$

where $I$ is the identity map, and

$$
\left(\nabla_{X}^{h} C_{T}\right) Y-\left(\nabla_{Y}^{h} C_{T}\right) X=C_{\nabla_{X}^{v} T} Y-C_{\nabla_{Y}^{v} T} X
$$

for any $S, T \in \Gamma(\mathcal{D})$ and $X, Y \in \Gamma\left(\mathcal{D}^{\perp}\right)$. For a proof we refer to [6] or [8].

\section{Elliptic submanifolds}

In this section, we recall from [7] the notion of elliptic submanifold of a space form as well as several of their basic properties.

Let $f: M^{m} \rightarrow \mathbb{Q}_{c}^{n}$ be an isometric immersion. The $\ell^{\text {th }}$-normal space $N_{\ell}^{f}(x)$ of $f$ at $x \in M^{m}$ for $\ell \geq 1$ is defined as

$$
N_{\ell}^{f}(x)=\operatorname{span}\left\{\alpha_{f}^{\ell+1}\left(X_{1}, \ldots, X_{\ell+1}\right): X_{1}, \ldots, X_{\ell+1} \in T_{x} M\right\} .
$$

Here $\alpha_{f}^{2}=\alpha_{f}$ and for $s \geq 3$ the so called $s^{\text {th }}$-fundamental form is the symmetric tensor $\alpha_{f}^{s}: T M \times \cdots \times T M \rightarrow N_{f} M$ defined inductively by

$$
\alpha_{f}^{s}\left(X_{1}, \ldots, X_{s}\right)=\pi^{s-1}\left(\nabla_{X_{s}}^{\perp} \cdots \nabla_{X_{3}}^{\perp} \alpha_{f}\left(X_{2}, X_{1}\right)\right)
$$

where $\pi^{k}$ stands for the projection onto $\left(N_{1}^{f} \oplus \cdots \oplus N_{k-1}^{f}\right)^{\perp}$.

An isometric immersion $f: M^{m} \rightarrow \mathbb{Q}_{c}^{n}$ of rank 2 is called elliptic if there exists a (necessary unique up to a sign) almost complex structure $J: \mathcal{D}^{\perp} \rightarrow \mathcal{D}^{\perp}$ such that the second fundamental form satisfies

$$
\alpha_{f}(X, X)+\alpha_{f}(J X, J X)=0
$$


for all $X \in \mathcal{D}^{\perp}$. Notice that $J$ is orthogonal if and only $f$ is minimal.

Let $f: M^{m} \rightarrow \mathbb{Q}_{c}^{n}$ be substantial and elliptic. The former means that the codimension cannot be reduced. Assume also that $f$ is nicely curved which means that for any $\ell \geq 1$ all subspaces $N_{\ell}^{f}(x)$ have constant dimension and thus form subbundles of the normal bundle. Notice that any $f$ is nicely curved along connected components of an open dense subset of $M^{m}$. Then, along that subset the normal bundle splits orthogonally and smoothly as

$$
N_{f} M=N_{1}^{f} \oplus \cdots \oplus N_{\tau_{f}}^{f}
$$

where all $N_{\ell}^{f}$ 's have rank 2, except possibly the last one that has rank 1 in case the codimension is odd. Thus, the induced bundle $f^{*} T \mathbb{Q}_{c}^{n}$ splits as

$$
f^{*} T \mathbb{Q}_{c}^{n}=f_{*} \mathcal{D} \oplus N_{0}^{f} \oplus N_{1}^{f} \oplus \cdots \oplus N_{\tau_{f}}^{f}
$$

where $N_{0}^{f}=f_{*} \mathcal{D}^{\perp}$. Setting

$$
\tau_{f}^{o}=\left\{\begin{array}{lll}
\tau_{f} & \text { if } n-m & \text { is even } \\
\tau_{f}-1 & \text { if } n-m & \text { is odd }
\end{array}\right.
$$

it turns out that the almost complex structure $J$ on $\mathcal{D}^{\perp}$ induces an almost complex structure $J_{\ell}$ on each $N_{\ell}^{f}, 0 \leq \ell \leq \tau_{f}^{o}$, defined by

$$
J_{\ell} \alpha_{f}^{\ell+1}\left(X_{1}, \ldots, X_{\ell}, X_{\ell+1}\right)=\alpha_{f}^{\ell+1}\left(X_{1}, \ldots, X_{\ell}, J X_{\ell+1}\right)
$$

where $\alpha_{f}^{1}=f_{*}$.

The $\ell^{\text {th }}$-order curvature ellipse $\mathcal{E}_{\ell}^{f}(x) \subset N_{\ell}^{f}(x)$ of $f$ at $x \in M^{m}$ for $0 \leq \ell \leq \tau_{f}^{o}$ is

$$
\mathcal{E}_{\ell}^{f}(x)=\left\{\alpha_{f}^{\ell+1}\left(Z_{\theta}, \ldots, Z_{\theta}\right): Z_{\theta}=\cos \theta Z+\sin \theta J Z \text { and } \theta \in[0, \pi)\right\}
$$

where $Z \in \mathcal{D}^{\perp}(x)$ has unit length and satisfies $\langle Z, J Z\rangle=0$. From ellipticity such a $Z$ always exists and $\mathcal{E}_{\ell}^{f}(x)$ is indeed an ellipse.

We say that the curvature ellipse $\mathcal{E}_{\ell}^{f}$ of an elliptic submanifold $f$ is a circle for some $0 \leq \ell \leq \tau_{f}^{o}$ if all ellipses $\mathcal{E}_{\ell}^{f}(x)$ are circles. That the curvature ellipse $\mathcal{E}_{\ell}^{f}$ is a circle is equivalent to the almost complex structure $J_{\ell}$ being orthogonal. Notice that $\mathcal{E}_{0}^{f}$ is a circle if and only if $f$ is minimal. An elliptic submanifold $f$ is called $\ell$-isotropic if all ellipses of curvature up to order $\ell$ are circles. Then $f$ is called isotropic if the ellipses of curvature of any order are circles.

Substantial isotropic surfaces in $\mathbb{R}^{2 n}$ are holomorphic curves in $\mathbb{C}^{n} \equiv \mathbb{R}^{2 n}$. Isotropic surfaces in spheres are also referred to as pseudoholomorphic surfaces. For this class of surfaces a Weierstrass type representation was given in [12]. 
Let $f: M^{m} \rightarrow \mathbb{Q}_{c}^{n-c},(c=0,1)$, be a substantial nicely curved elliptic submanifold. Assume that $M^{m}$ is the saturation of a fixed simply connected cross section $L^{2} \subset M^{m}$ to the relative nullity foliation. The subbundles in the orthogonal splitting (4) are parallel in the normal connection (and thus in $\mathbb{Q}_{c}^{n-c}$ ) along $\mathcal{D}$. Hence each $N_{\ell}^{f}$ can be seen as a vector bundle along the surface $L^{2}$.

A polar surface to $f$ is an immersion $h$ of $L^{2}$ defined as follows:

(a) If $n-c-m$ is odd, then the polar surface $h: L^{2} \rightarrow \mathbb{S}^{n-1}$ is the spherical image of the unit normal field spanning $N_{\tau_{f}}^{f}$.

(b) If $n-c-m$ is even, then the polar surface $h: L^{2} \rightarrow \mathbb{R}^{n}$ is any surface such that $h_{*} T_{x} L=N_{\tau_{f}}^{f}(x)$ up to parallel identification in $\mathbb{R}^{n}$.

Polar surfaces always exist since in case (b) any elliptic submanifold admits locally many polar surfaces.

The almost complex structure $J$ on $\mathcal{D}^{\perp}$ induces an almost complex structure $\tilde{J}$ on $T L$ defined by $P \tilde{J}=J P$ where $P: T L \rightarrow \mathcal{D}^{\perp}$ is the orthogonal projection. It turns out that a polar surface of an elliptic submanifold is necessarily elliptic. Moreover, if the elliptic submanifold has a circular ellipse of curvature then its polar surface has the same property at the "corresponding" normal bundle. As a matter of fact, up to parallel identification it holds that

$$
N_{s}^{h}=N_{\tau_{f}^{o}-s}^{f} \text { and } J_{s}^{h}=\left(J_{\tau_{f}^{o}-s}^{f}\right)^{t}, \quad 0 \leq s \leq \tau_{f}^{o} .
$$

In particular, the polar surface is nicely curved. Notice that the last $\ell+1$ ellipses of curvature of the polar surface to an $\ell$-isotropic submanifold are circles. Note that in this case the polar surface is not necessarily minimal.

A bipolar surface to $f$ is any polar surface to a polar surface to $f$. In particular, if we are in case $f: M^{3} \rightarrow \mathbb{S}^{n-1}$, then a bipolar surface to $f$ is a nicely curved elliptic immersion $g: L^{2} \rightarrow \mathbb{R}^{n}$.

\section{The local case}

We discuss next two alternative ways to parametrically describe, at least locally, all spherical three-dimensional minimal submanifolds of rank two in spheres. This follows from the results in [7] bearing in mind that a submanifold is minimal in a sphere if and only if the cone shaped over it is minimal in the Euclidean space.

Let $g: L^{2} \rightarrow \mathbb{R}^{n+1}, n \geq 4$, be an elliptic surface and let $T^{1} L$ denote its unit tangent bundle. 
Proposition 4. If $\mathcal{E}_{1}^{g}$ is a circle, then the map $\psi_{g}: T^{1} L \rightarrow \mathbb{S}^{n}$ given by

$$
\psi_{g}(x, w)=g_{*} w
$$

is a minimal immersion with index of relative nullity $\nu \geq 1$ outside the subset of singular points, which correspond to points where $\operatorname{dim} N_{1}^{g}=0$. Moreover, a regular point $(x, w) \in T^{1} L$ is totally geodesic for $\psi_{g}$ if and only if $\operatorname{dim} N_{2}^{g}(x)=0$. Conversely, any three-dimensional minimal submanifold in the sphere with $\nu=1$ at any point can be at least locally parametrized in this way.

The above parametrization (used for Theorem 1) is called the bipolar parametrization in [7] because $g$ is a bipolar surface to $\psi_{g}$. The parametrization in the sequel (used for the examples discussed above) was called in [7] the polar parametrization.

Let $g: L^{2} \rightarrow \mathbb{Q}_{1-c}^{2 n+2 c}(c=0,1), n \geq 2$, be a nicely curved elliptic surface and let $M^{3}=U N_{\tau_{g}}^{g}$ stand for the unit subbundle of $N_{\tau_{g}}^{g}$.

Proposition 5. If $\mathcal{E}_{\tau_{g}-1}^{g}$ is a circle, then $\phi_{g}: M^{3} \rightarrow \mathbb{S}^{2 n+c}$ given by $\phi_{g}(x, w)=w$ is a minimal immersion of rank two and polar surface $g$. Conversely, any minimal submanifold $M^{3}$ in $\mathbb{S}^{2 n+c}$ of rank two can locally be parametrized in this way.

\section{The complete case}

We first observe that for complete submanifolds of rank at most two the interesting case is the three-dimensional one. The remaining of the paper is devoted to the study of the latter case.

Proposition 6. Let $f: M^{m} \rightarrow \mathbb{S}^{n}, m \geq 3$, be a minimal isometric immersion with index of relative nullity $\nu \geq m-2$ at any point. If $M^{m}$ is complete, then $f$ is totally geodesic unless $m=3$.

The above is an immediate consequence of the following result due to Ferus [17] (see [6, Lemma 6.16] where the proof holds regardless the codimension) since due to minimality we cannot have points with index of relative nullity $m-1$.

Lemma 7. Let $f: M^{m} \rightarrow \mathbb{S}^{n}$ be an isometric immersion and let $U \subset M^{m}$ be an open subset where the index of relative nullity is constant either $\nu=m-1$ or $\nu=m-2$. Then no leaf of relative nullity contained in $U$ is complete if $m \geq 4$.

In the sequel, let $f: M^{3} \rightarrow \mathbb{S}^{n}$ be a minimal isometric immersion of a complete Riemannian manifold with index of relative nullity $\nu(x) \geq 1$ at any $x \in M^{3}$. Let $U \subset M^{3}$ be an open subset where $\nu=1$ such that $\mathcal{D}$ is a line bundle on $U$. If that line bundle is trivial, then there is a unique, up to a sign, orthogonal almost complex structure $J:\left.\left.\mathcal{D}^{\perp}\right|_{U} \rightarrow \mathcal{D}^{\perp}\right|_{U}$. In that case set $\mathcal{C}=C_{e}$ where $e$ is a unit section of $\left.\mathcal{D}\right|_{U}$. 
Lemma 8. If $\left.\mathcal{D}\right|_{U}$ is a trivial line bundle there are harmonic functions $u, v \in C^{\infty}(U)$ such that

$$
\mathcal{C}=v I-u J
$$

Proof: The proof follows similarly as in [10]. Without loss of generality we assume that $f$ is substantial. Denote by $A_{\xi}$ the shape operator of $f$ with respect to $\xi \in N_{f} M$. The Codazzi equation gives

$$
\left.\nabla_{e} A_{\xi}\right|_{\mathcal{D}^{\perp}}=\left.A_{\xi}\right|_{\mathcal{D}^{\perp}} \circ \mathcal{C}+\left.A_{\nabla_{e}^{\perp} \xi}\right|_{\mathcal{D}^{\perp}}
$$

for any vector field $\xi \in N_{f} M$. In particular,

$$
\left.A_{\xi}\right|_{\mathcal{D}^{\perp}} \circ \mathcal{C}=\left.\mathcal{C}^{t} \circ A_{\xi}\right|_{\mathcal{D}^{\perp}}
$$

Moreover, the minimality condition is equivalent to

$$
\left.A_{\xi}\right|_{\mathcal{D}^{\perp}} \circ J=\left.J^{t} \circ A_{\xi}\right|_{\mathcal{D}^{\perp}}
$$

First we consider the case $n=4$. Let $e_{1}, e_{2}, e_{3}=e$ be a local orthonormal frame field that diagonalizes $A_{\xi}$ with respect to a unit normal vector filed $\xi$ such that $J e_{1}=e_{2}$. Set

$$
u=\left\langle\nabla_{e_{2}} e_{1}, e_{3}\right\rangle \text { and } v=\left\langle\nabla_{e_{1}} e_{1}, e_{3}\right\rangle
$$

From the Codazzi equations

$$
\left(\nabla_{e_{i}} A_{\xi}\right) e_{3}=\left(\nabla e_{3} A_{\xi}\right) e_{i} \quad 1 \leq i \leq 2
$$

we obtain that $\left\langle\nabla_{e_{2}} e_{2}, e_{3}\right\rangle=v$ and from the Codazzi equation

$$
\left\langle\left(\nabla_{e_{1}} A_{\xi}\right) e_{2}, e_{3}\right\rangle=\left\langle\left(\nabla_{e_{2}} A_{\xi}\right) e_{1}, e_{3}\right\rangle
$$

that $\left\langle\nabla_{e_{1}} e_{2}, e_{3}\right\rangle=-u$, and now (6) follows.

Assume now that $f$ does not reduce codimension to one. Due to the minimality assumption, we have that $\operatorname{dim} N_{1}^{f} \leq 2$. If $\operatorname{dim} N_{1}^{f}=1$ on an open subset $V \subset M^{3}$, a simple argument using the Codazzi equation gives that $N_{1}^{f}$ is parallel in the normal bundle, and hence $\left.f\right|_{V}$ reduces codimension to one. Due to real analyticity the same would hold globally, and that has been excluded. Hence, there is an open dense subset $W \subset M^{3}$ where $\operatorname{dim} N_{1}^{f}=2$. From (17) and (8) we have that $\mathcal{C} \in \operatorname{span}\{I, J\}$ on $U \cap W$, and (6) follows easily.

We now show that $u$ and $v$ are harmonic functions. From (2) and (3) we have

$$
\nabla_{e}^{h} \mathcal{C}=\mathcal{C}^{2}+I
$$

and

$$
\left(\nabla_{X}^{h} \mathcal{C}\right) Y=\left(\nabla_{Y}^{h} \mathcal{C}\right) X
$$


for any $X, Y \in \mathcal{D}^{\perp}$. Let $e_{1}, e_{2}, e_{3}$ be a local orthonormal frame with $J e_{1}=e_{2}$ and $e_{3} \in \mathcal{D}$. From ([6) we find that

$$
v=\left\langle\nabla_{e_{1}} e_{1}, e_{3}\right\rangle=\left\langle\nabla_{e_{2}} e_{2}, e_{3}\right\rangle
$$

and

$$
u=-\left\langle\nabla_{e_{1}} e_{2}, e_{3}\right\rangle=\left\langle\nabla_{e_{2}} e_{1}, e_{3}\right\rangle
$$

Equation (9) is equivalent to

$$
e_{3}(v)=v^{2}-u^{2}+1 \text { and } e_{3}(u)=2 u v
$$

whereas (10) is equivalent to

$$
e_{1}(u)=e_{2}(v) \text { and } e_{2}(u)=-e_{1}(v) .
$$

The Laplacian of $v$ is given by

$$
\Delta v=\sum_{i=1}^{3} e_{i} e_{i}(v)-\omega_{12}\left(e_{1}\right) e_{2}(v)-\omega_{13}\left(e_{1}\right) e_{3}(v)+\omega_{12}\left(e_{2}\right) e_{1}(v)-\omega_{23}\left(e_{2}\right) e_{3}(v)
$$

where $\omega_{i j}\left(e_{k}\right)=\left\langle\nabla_{e_{k}} e_{i}, e_{j}\right\rangle$ for any $i, j, k \in\{1,2,3\}$. Using (14) we have

$$
\begin{aligned}
e_{1} e_{1}(v)+e_{2} e_{2}(v) & =-e_{1} e_{2}(u)+e_{2} e_{1}(u)=-\left[e_{1}, e_{2}\right](u)=-\nabla_{e_{1}} e_{2}(u)+\nabla_{e_{2}} e_{1}(u) \\
& =\omega_{12}\left(e_{1}\right) e_{1}(u)-\omega_{23}\left(e_{1}\right) e_{3}(u)+\omega_{12}\left(e_{2}\right) e_{2}(u)+\omega_{13}\left(e_{2}\right) e_{3}(u) \\
& =\omega_{12}\left(e_{1}\right) e_{2}(v)-\omega_{12}\left(e_{2}\right) e_{1}(v)+2 u e_{3}(u) .
\end{aligned}
$$

Inserting this equality into the previous equation and making use of (11) and (13) yields $\Delta v=0$. In a similar form it follows that also $u$ is harmonic.

Let $\mathcal{A}$ denote the set of totally geodesic points of $f$. By Proposition 3 the relative nullity distribution $\mathcal{D}$ is a line bundle on $M^{3} \backslash \mathcal{A}$. Being $f$ real analytic, the square of the norm of the second fundamental form is a real analytic function and hence $\mathcal{A}$ is a real analytic set. According to Lojasewicz's structure theorem [20, Theorem 6.3.3] the set $\mathcal{A}$ locally decomposes as

$$
\mathcal{A}=\mathcal{V}^{0} \cup \mathcal{V}^{1} \cup \mathcal{V}^{2} \cup \mathcal{V}^{3}
$$

where each $\mathcal{V}^{d}, 0 \leq d \leq 3$, is either empty or a disjoint finite union of $d$-dimensional real analytic subvarieties. A point $x_{0} \in \mathcal{A}$ is called a regular point of dimension $d$ if there is a neighborhood $\Omega$ of $x_{0}$ such that $\Omega \cap \mathcal{A}$ is a $d$-dimensional real analytic submanifold of $\Omega$. Otherwise $x_{0}$ is said to be a singular point. The set of singular points is locally a finite union of submanifolds.

We want to show that $\mathcal{A}=\mathcal{V}^{1}$ unless $f$ is just a totally geodesic three-sphere in $\mathbb{S}^{n}$. After excluding the latter case, we have from the real analyticity of $f$ that $\mathcal{V}^{3}$ is empty. We will proceed now following ideas developed in [10]. In fact, we only sketch the proof of the following fact, which is similar to the proof of Lemma 2 in [10]. 
Lemma 9. The set $\mathcal{V}^{2}$ is empty.

Proof: We only have to show that there are no regular points in $\mathcal{V}^{2}$. Suppose that a regular point $x_{0} \in \mathcal{V}^{2}$ exists. Let $\Omega \subset M^{3}$ be an open neighborhood of $x_{0}$ such that $L^{2}=\Omega \cap \mathcal{A}$ is an embedded surface. Let $e_{1}, e_{2}, e_{3}, \xi_{1}, \ldots, \xi_{n-3}$ be an orthonormal frame adapted to $M^{3}$ along $\Omega$ near $x_{0}$. The coefficients of the second fundamental form are $h_{i j}^{a}=\left\langle\alpha_{f}\left(e_{i}, e_{j}\right), \xi_{a}\right\rangle$ where $1 \leq i, j, k \leq 3$ and $1 \leq a, b \leq n-3$.

The Gauss map $\gamma: M^{3} \rightarrow \operatorname{Gr}(4, n+1)$ of $f$ is a map into the Grassmannian of oriented 4-dimensional subspaces in $\mathbb{R}^{n+1}$ defined by

$$
\gamma=f \wedge e_{1} \wedge e_{2} \wedge e_{3}
$$

where we regard $\operatorname{Gr}(4, n+1)$ as a submanifold in $\wedge^{4} \mathbb{R}^{n+1}$ via the map for the Plücker embedding. Then

$$
\gamma_{*} e_{i}=\sum_{j, a} h_{i j}^{a} f \wedge e_{j a}
$$

where $e_{j a}$ is taken by replacing $e_{j}$ with $\xi_{a}$ in $e_{1} \wedge e_{2} \wedge e_{2}$. Moreover, it easy to see that the Gauss map satisfies the partial differential equation

$$
\Delta \gamma+\left\|\alpha_{f}\right\|^{2} \gamma=\sum_{i, a \neq b, j \neq k} h_{i j}^{a} h_{i k}^{b} f \wedge e_{j a, k b}
$$

where $e_{j a, k b}$ is obtained by replacing $e_{j}$ with $\xi_{a}$ and $e_{k}$ with $\xi_{b}$ in $e_{1} \wedge e_{2} \wedge e_{3}$. Hence, we may write the latter equation in the form

$$
\Delta \gamma(x)+\left\|\gamma_{*}(x)\right\|^{2} \gamma(x)+G\left(x, \gamma_{*}\right)=0
$$

where $G$ is real analytic with $G(\cdot, 0)=0$. Clearly, we have that $\gamma$ is constant along $L^{2}$ and that $\gamma_{*}(\mathbf{n})=0$ on $L^{2}$, where $\mathbf{n}$ is a unit normal of $L^{2} \subset M^{3}$. Then, it follows from the uniqueness part of the Cauchy-Kowalewsky theorem (cf. [23]) that the Gauss map $\gamma$ must be constant. This would imply that $f(M)$ is a three-dimensional totally geodesic sphere which contradicts our assumption.

Lemma 10. The set $\mathcal{V}^{0}$ is empty.

Proof: Let $\Omega$ be an open neighborhood around $x_{0} \in \mathcal{V}^{0}$ such that $\nu=1$ on $\Omega \backslash\left\{x_{0}\right\}$ and let $\left\{x_{j}\right\}_{j \in \mathbb{N}}$ be a sequence in $\Omega \backslash\left\{x_{0}\right\}$ converging to $x_{0}$. Let $e_{j}=e\left(x_{j}\right) \in T_{x_{j}} M$ be the sequence of unit vectors contained in the relative nullity distribution of $f$. By passing to a subsequence, if necessary, there is a unit vector $e_{0} \in T_{x_{0}} M$ such that $\lim e_{j}=e_{0}$. By continuity, the geodesic tangent to $e_{0}$ at $x_{0}$ is a leaf of relative nullity outside $x_{0}$. But this is a contradiction in view of Proposition 3 ,

Lemma 11. The foliation of relative nullity extends analytically over the regular points in the set $\mathcal{A}$. 
Proof: Because $\mathcal{A}=\mathcal{V}^{1}$ its 2-capacity $\operatorname{cap}_{2}(\mathcal{A})$ must be zero (cf. [16, Theorem 3]). On the other hand, the distribution $\mathcal{D}$ extends continuously over the regular points of $\mathcal{A}$. In fact, by the previous lemmas it remains to consider the case when $\Omega$ is an open subset of $M^{3}$ such that $\Omega \cap \mathcal{A}$ is a open piece of a great circle in the ambient space. But in this situation the result follows by an argument of continuity similar than in the proof of Lemma 10.

Let $\Omega$ be an open subset of $M^{3} \backslash \mathcal{A}$ and $e_{1}, e_{2}, e_{3}$ a local frame on $\Omega$ as in the proof of Lemma 8. Consider the map $F: \Omega \rightarrow \mathbb{S}^{n}$ with values into the unit sphere given by $F=f_{*} e_{3}$. A straightforward computation using (11), (12) and (14) shows that its tension field

$$
\tau(F)=\sum_{j=1}^{3}\left(\bar{\nabla}_{F_{*} e_{j}} F_{*} e_{j}-F_{*} \nabla_{e_{j}} e_{j}\right)
$$

vanishes, where $\bar{\nabla}$ denotes the Levi-Civita connection of $\mathbb{S}^{n}$. Hence $F$ is a harmonic

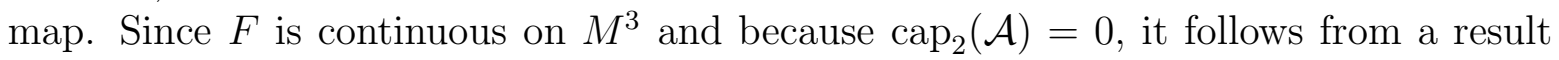
of Meier [22, Theorem 1] that $F$ is of class $C^{2}$ on $M^{3}$. But then $F$ is real analytic by a result due to Eells and Sampson [14, Proposition p. 117].

Lemma 12. The set $\mathcal{A}$ has no singular points.

Proof: Let $x_{0} \in \mathcal{A}$ be a singular point. From Lemmas 9 and 10 the set $\mathcal{A}$ contains subvarieties of dimension one. It is well known that the singular points of such curves are isolated (cf. [20, Theorem 6.3.3]). Moreover, according to Lemma 11 the set of regular points of $\mathcal{A}$ contains geodesic curves of the relative nullity foliation. Hence $x_{0}$ is an intersection of such geodesic curves. Let $\Omega \subset M^{3}$ be an open subset containing $x_{0}$ such that the restriction of $\left.f\right|_{\Omega}$ is injective. Consider a fixed cross section $L^{2}$ to $\mathcal{D}$ on $\Omega \backslash\left\{x_{0}\right\}$. Note that the immersion $f$ can be locally parametrized by the embedding $\phi: L^{2} \times \mathbb{S}^{1} \rightarrow \mathbb{S}^{n}$ given by

$$
\phi(x, t)=\exp _{f(x)}\left(t f_{*} e\right)=\cos t f(x)+\sin t f_{*} e(x)
$$

where $\left.e \in \mathcal{D}\right|_{L^{2}}$. Since $x_{0}$ is an intersection point of geodesics in the relative nullity foliation, it follows from the parametrization that there are points $\left(x_{1}, t_{1}\right),\left(x_{2}, t_{2}\right) \in$ $L^{2} \times \mathbb{S}^{1}$ such that

$$
\phi\left(x_{1}, t_{1}\right)=f\left(x_{0}\right)=\phi\left(x_{2}, t_{2}\right),
$$

which leads to a contradiction.

\section{The proofs}

The proof of our main result relies heavily on the following consequence of the OmoriYau maximum principle. 
Lemma 13. Let $M^{m}$ be a Riemannian manifold for which the Omori-Yau maximum principle holds. If $\varphi \in C^{\infty}(M)$ satisfies the partial differential inequality $\Delta \varphi \geq 2 \varphi^{2}$, then $\sup \varphi=0$. In particular, if $\varphi \geq 0$ then $\varphi=0$.

Proof: See [1, Theorem 2.8] or [18].

Proof of Theorem 1: By Proposition 6] we only have to consider the case $m=3$. We distinguish two cases.

Case $\mathcal{A}=\emptyset$. At first suppose that the line bundle $\mathcal{D}$ is trivial with $e$ a unit global section. By Lemma 8 there are harmonic functions $u, v \in C^{\infty}(M)$ such that $\mathcal{C}=v I-u J$.

We claim that $u$ is nowhere zero. To the contrary suppose that $u\left(x_{0}\right)=0$ at $x_{0} \in M^{3}$. Let $\gamma: \mathbb{R} \rightarrow M^{3}$ the maximal integral curve of $e$ emanating from $x_{0}$. The second equation in (13) gives that $u$ must vanish along $\gamma$. Thus the first equation in (13) reduces to $v^{\prime}(s)=v^{2}(s)+1$, where $v(s)=v(\gamma(s))$ is an entire function. But this is a contradiction since this equation has no entire solutions. In the sequel we assume that $u>0$.

Using (13) one can easily see that

$$
\begin{aligned}
\Delta\left((u-1)^{2}+v^{2}\right) & =2\left(\|\nabla u\|^{2}+\|\nabla v\|^{2}\right) \geq 2\left((e(u))^{2}+(e(v))^{2}\right) \\
& \geq 2\left((u-1)^{2}+v^{2}\right)^{2},
\end{aligned}
$$

and Lemma 13 implies that $\mathcal{C}=-J$.

Let $\mathcal{U} \subset M^{3}$ be the open dense subset where $f$ is nicely curved. Then let $U \subset \mathcal{U}$ be an open connected subset of $\mathcal{U}$ that is the saturation of a simply connected cross section $L^{2} \subset U$ to the relative nullity foliation. Hereafter we work on $U$ where $f$ is nicely curved. Hence polar and bipolar surfaces of $\left.f\right|_{U}$ are well defined.

Let $h$ be a polar surface to $\left.f\right|_{U}$. We have seen that the almost complex structure $J$ on $\mathcal{D}^{\perp}$ induces an almost complex structure $\tilde{J}$ on $T L$ defined by $P \tilde{J}=J P$, where $P: T L \rightarrow \mathcal{D}^{\perp}$ is the orthogonal projection. Moreover, $h$ is elliptic with respect to $\tilde{J}$ and (5) holds. In addition, it follows from Proposition 5 that $\mathcal{E}_{\tau_{h}-1}^{h}$ is a circle.

We claim that the last curvature ellipse $\mathcal{E}_{\tau_{h}}^{h}$ of $h$ is also a circle. In that case the bipolar surface $g: L^{2} \rightarrow \mathbb{R}^{n+1}$ to $f$ is 1-isotropic, and we are done. Observe that

$$
N_{\tau_{h}}^{h}=\operatorname{span}\{\xi, \eta\}
$$

where $\xi=\left.f_{*} e\right|_{L^{2}}$ and $\eta=\left.f\right|_{L^{2}}$. Using $\mathcal{C}=-J$, we obtain that

$$
\xi_{*}=\left.f_{*}\right|_{\mathcal{D}^{\perp}} \circ J \circ P .
$$

Consider vector fields $X_{1}, \ldots, X_{\tau_{h}}, Y \in T L$. Since $N_{\tau_{h}-1}^{h}=N_{0}^{f}=f_{*}\left(\mathcal{D}^{\perp}\right)$, we have

$$
\alpha_{h}^{\tau_{h}}\left(X_{1}, \ldots, X_{\tau_{h}}\right)=f_{*} Z
$$


for some $Z \in \mathcal{D}^{\perp}$. Keeping in mind the bundle isometries, we obtain that

$$
\alpha_{h}^{\tau_{h}+1}\left(X_{1}, \ldots, X_{\tau_{h}}, Y\right)=\left(\nabla_{Y}^{h \perp} \alpha_{h}^{\tau_{h}}\left(X_{1}, \ldots, X_{\tau_{h}}\right)\right)_{N_{\tau_{h}}^{h}}=\left(\tilde{\nabla}_{Y} f_{*} Z\right)_{N_{\tau_{h}}^{h}} .
$$

Taking into account (15) we see that

$$
\begin{aligned}
\alpha_{h}^{\tau_{h}+1}\left(X_{1}, \ldots, X_{\tau_{h}}, Y\right) & =\left\langle\tilde{\nabla}_{Y} f_{*} Z, \xi\right\rangle \xi+\left\langle\tilde{\nabla}_{Y} f_{*} Z, \eta\right\rangle \eta \\
& =-\left\langle f_{*} Z, \xi_{*} Y\right\rangle \xi-\left\langle f_{*} Z, \eta_{*} Y\right\rangle \eta \\
& =-\langle Z, J P Y\rangle \xi-\langle Z, P Y\rangle \eta
\end{aligned}
$$

Recall that the almost complex structure $J_{\tau_{h}}^{h}$ on $N_{\tau_{h}}^{h}$ is given by

$$
J_{\tau_{h}}^{h} \alpha_{h}^{\tau_{h}+1}\left(X_{1}, \ldots, X_{\tau_{h}}, Y\right)=\alpha_{h}^{\tau_{h}+1}\left(X_{1}, \ldots, X_{\tau_{h}}, \tilde{J} Y\right)
$$

Since

$$
\alpha_{h}^{\tau_{h}+1}\left(X_{1}, \ldots, X_{\tau_{h}}, Y\right)=-\langle Z, J P Y\rangle \xi-\langle Z, P Y\rangle \eta
$$

and

$$
\alpha_{h}^{\tau_{h}+1}\left(X_{1}, \ldots, X_{\tau_{h}}, \tilde{J} Y\right)=\langle Z, P Y\rangle \xi-\langle Z, J P Y\rangle \eta
$$

we have that the vectors $\alpha_{h}^{\tau_{h}+1}\left(X_{1}, \ldots, X_{\tau_{h}}, Y\right)$ and $\alpha_{h}^{\tau_{h}+1}\left(X_{1}, \ldots, X_{\tau_{h}}, \tilde{J} Y\right)$ are perpendicular of the same length. Thus $J_{\tau_{h}}^{h}$ is orthogonal, and proves the claim.

Finally, if the line bundle $\mathcal{D}$ is not trivial, it suffices to argue for a 2-fold covering $\Pi: \tilde{M}^{3} \rightarrow M^{3}$ such that the nullity distribution $\tilde{\mathcal{D}}$ of $\tilde{f}=f \circ \Pi$ is a trivial line bundle and $\Pi_{*} \tilde{\mathcal{D}}=\mathcal{D}$.

Case $\mathcal{A} \neq \emptyset$. We have seen that the relative nullity distribution $\mathcal{D}$ can be extended analytically to a line bundle on $M^{3}$, denoted again by $\mathcal{D}$, over the set of totally geodesic points $\mathcal{A}$. Without loss of generality, we may assume that there is a global unit section $e \in \mathcal{D}$, since otherwise we can pass to the 2 -fold covering space

$$
\tilde{M}^{3}=\left\{(x, w): x \in M^{3}, w \in \mathcal{D}(x) \text { and }\|w\|=1\right\}
$$

and argue as in the previous case. From Lemma 8, we know that there exist harmonic functions $u, v \in C^{\infty}\left(M^{3} \backslash \mathcal{A}\right)$ such that (6) holds on $M^{3} \backslash \mathcal{A}$. By previous results the functions $u$ and $v$ can be extended analytically to harmonic functions on the entire $M^{3}$. Moreover, since $u$ is positive on $M^{3} \backslash \mathcal{A}$ and $\mathcal{A}$ consists of geodesic curves, by continuity we get that $u \geq 0$ on $M^{3}$. Then $\|\mathcal{C}+J\|^{2}$ is globally well defined and, arguing as in the previous case, we conclude again that $\mathcal{C}=-J$ on $M^{3}$. The remaining of the proof now goes as before.

Proof of Corollary [2: By a result of Ejiri [15] we have that $f$ is minimal. Let $e_{1}, e_{2}, e_{3}$ be a local orthonormal tangent frame such that $e_{3} \in \mathcal{D}$. Since $f$ is Lagrangian, we have 
that $J e_{1}, J e_{2}, J e_{3}$ is an orthonormal frame in the normal bundle of $f$. Moreover, it is well known that the 3 -linear tensor $h$ given by

$$
h\left(e_{i}, e_{j}, e_{k}\right)=\left\langle\alpha_{f}\left(e_{i}, e_{j}\right), J e_{k}\right\rangle, \quad i, j, k \in\{1,2,3\},
$$

is fully symmetric. Away from the totally geodesic points, it is easy to check that the vector fields $\alpha_{f}\left(e_{1}, e_{1}\right)$ and $\alpha_{f}\left(e_{1}, e_{2}\right)$ are perpendicular to each other and have the same length. Hence $\mathcal{E}_{1}^{f}$ is a circle.

Suppose at first that $f$ is substantial in $\mathbb{S}^{6}$. Assume that the submanifold is the saturation of a fixed cross section $L^{2}$ to the relative nullity foliation. Let $h: L^{2} \rightarrow \mathbb{S}^{6}$ be a polar surface to $f$. From (5) we obtain that $h$ is 1 -isotropic. Proceeding as in the proof of Theorem 1, we deduce that the second ellipse of $h$ is also a circle. Therefore, $h$ is pseudoholomorphic and any bipolar surface $g$ to $f$ is 2-isotropic in $\mathbb{R}^{7}$.

Now we consider the case where $f$ is substantial in $\mathbb{S}^{5}$. Take a fixed cross section $L^{2}$ to the relative nullity foliation and let $h: L^{2} \rightarrow \mathbb{R}^{6}$ be a polar surface to $f$. As in the previous case, we obtain that $h$ must be isotropic. Therefore, any bipolar surface $g$ to $f$ is an isotropic surface in $\mathbb{R}^{6}$.

\section{References}

[1] L. Alias, P. Mastrolia and M. Rigoli, "Maximum principles and geometric applications". Springer Monographs in Mathematics. Springer, Cham, 2016.

[2] J. Barbosa, On minimal immersions of $\mathbb{S}^{2}$ into $\mathbb{S}^{2 m}$. Trans. Amer. Math. Soc. 210 (1975), 75-106.

[3] R. Bryant, Some remarks on the geometry of austere manifolds. Bol. Soc. Brasil. Mat. 21 (1991), 133-157.

[4] E. Calabi, Minimal immersions of surfaces in Euclidean spheres. J. Differential Geom. 1 (1967), 111-125.

[5] B. Y. Chen "Pseudo-Riemannian geometry, $\delta$-invariants and applications". World Scientific Publishing Co. Hackensack, NJ, 2011.

[6] M. Dajczer et al., "Submanifolds and Isometric Immersions", Math. Lecture Ser. 13, Publish or Perish Inc. Houston, 1990.

[7] M. Dajczer and L. Florit, A class of austere submanifolds. Illinois J. Math. 45 (2001), $735-755$.

[8] M. Dajczer and D. Gromoll, Rigidity of complete Euclidean hypersurfaces. J. Differential Geom. 31 (1990), 401-416. 
[9] M. Dajczer and D. Gromoll, The Weierstrass representation for complete minimal real Kaehler submanifolds of codimension two. Invent. Math. 119 (1995), 235-242.

[10] M. Dajczer, Th. Kasioumis, A. Savas-Halilaj and Th. Vlachos, Complete minimal submanifolds with nullity in Euclidean space, to appear in Math. Z.

[11] M. Dajczer and Th. Vlachos, The associated family of an elliptic surface and an application to minimal submanifolds. Geom. Dedicata 178 (2015), 259-275.

[12] M. Dajczer and Th. Vlachos, A representation for pseudoholomorphic surfaces in spheres. Proc. Amer. Math. Soc. 144 (2016), 3105-3113.

[13] F. Dillen and L. Vrancken, Totally real submanifolds in $\mathbb{S}^{6}$ satisfying Chen's equality. Trans. Amer. Math. Soc. 348 (1996), 1633-1646.

[14] J. Eells and J. Sampson, Harmonic mappings of Riemannian manifolds. Amer. J. Math. 86 (1964), 109-160.

[15] N. Ejiri, Totally real submanifolds in a 6-sphere. Proc. Amer. Math. Soc. 83 (1981), 759-763.

[16] L. Evans and R. Gariepy, "Measure Theory and Fine Properties of Functions". Studies in Advanced Mathematics. CRC Press, Boca Raton (1992).

[17] D. Ferus, The rigidity of complete hypersurfaces, Unpublished.

[18] Th. Hasanis, A. Savas-Halilaj and Th. Vlachos. Complete minimal hypersurfaces in the hyperbolic space $\mathbb{H}^{4}$ with vanishing Gauss-Kronecker curvature. Trans. Amer. Math. Soc. 359 (2007), 2799-2818.

[19] R. Harvey and B. Lawson, Calibrated geometries. Acta Math. 148 (1982), 17-157.

[20] S. Krantz and H. Parks, "A primer of real analytic functions". Birkhäuser Advanced Texts: Basler Lehrbücher, Birkhäuser Boston, Inc., Boston, MA, 2002.

[21] J. Lotay, Associative submanifolds of the 7-sphere. Proc. Lond. Math. Soc. 105 (2012), 1183-1214.

[22] M. Meier, Removable singularities of harmonic maps and an application to minimal submanifolds. Indiana Univ. Math. J. 35 (1986), 705-726.

[23] M. Taylor, "Partial differential equations I. Basic theory". Second edition. Applied Mathematical Sciences, 115. Springer, New York, 2011. 


\author{
Marcos Dajczer \\ IMPA - Estrada Dona Castorina, 110 \\ 22460-320, Rio de Janeiro - Brazil \\ e-mail: marcos@impa.br \\ Theodoros Kasioumis \\ University of Ioannina \\ Department of Mathematics \\ Ioannina-Greece \\ e-mail: theokasio@gmail.com \\ Andreas Savas-Halilaj \\ Leibniz Universität Hannover \\ Institut für Differentialgeometrie \\ Welfengarten 1 \\ 30167 Hannover-Germany \\ e-mail: savasha@math.uni-hannover.de \\ Theodoros Vlachos \\ University of Ioannina \\ Department of Mathematics \\ Ioannina-Greece \\ e-mail: tvlachos@uoi.gr
}

